\title{
Which level is responsible for gluteal pain in lumbar disc hernia?
}

\author{
Guofang Fang ${ }^{1,2}$, Jianhe Zhou ${ }^{3}$, Yutan Liư ${ }^{4}$, Hongxun Sang ${ }^{2}$, Xiangyang $X u^{3}$ and Zihai Ding ${ }^{{ }^{*}}$
}

\begin{abstract}
Background: There are many different reasons why patients could be experiencing pain in the gluteal area. Previous studies have shown an association between radicular low back pain (LBP) and gluteal pain (GP). Studies locating the specific level responsible for gluteal pain in lumbar disc hernias have rarely been reported.

Methods: All patients with lumbar disc herniation (LDH) in the Kanghua hospital from 2010 to 2014 were recruited. All patients underwent a lumbar spine MRI to clarify their LDH diagnosis, and patients were allocated to a GP group and a non-GP group. To determine the cause and effect relationship between LDH and GP, all of the patients were subjected to percutaneous endoscopic lumbar discectomy (PELD).
\end{abstract}

Results: A total of 286 cases were included according to the inclusive criteria, with 168 cases in the GP group and 118 cases in the non-GP group. Of these, in the GP group, 159 cases involved the L4/5 level and 9 cases involved the L5/S1 level, while in the non-GP group, 43 cases involved the L4/5 level and 48 cases involved the L5/S1 level. PELD was performed in both groups. Gluteal pain gradually disappeared after surgery in all of the patients. Gluteal pain recrudesced in a patient with recurrent disc herniation (L4/5).

Conclusions: As a clinical finding, gluteal pain is related to low lumbar disc hernia. The $L 4 / 5$ level is the main level responsible for gluteal pain in lumbar disc hernia. No patients with gluteal pain exhibited involvement at the L3/4 level.

Keyword: LBP, Gluteal pain, LDH, Percutaneous endoscopic lumbar discectomy

Abbreviations: GP, Gluteal Pain; LBP, Low Back Pain; LDH, Lumbar Disc Herniation; PELD, Percutaneous Endoscopic Lumbar Discectomy; SCNEN, Superior Cluneal Nerve Entrapment Neuropathy

\section{Background}

Among people suffer from low back pain (LBP) during their life time [1], for $80 \%$, their regular activity was limited due to LDH [2]. Paraspinal region pain is the most commonly encountered pain in lumbar disc hernia. Gluteal pain is also a complaint among some patients with $\mathrm{LDH}$ and is sometimes the only complaint. Approximately three-quarters of patients with unilateral radicular pain presented with gluteal pain [3].

However, in clinical practice, complaints of gluteal and radicular pain in the affected leg have always been diagnosed as deep gluteal syndrome (DGS). DGS is a condition in which the sciatic nerve is compressed by any structures in the deep gluteal space. The chief symptoms of DGS are

\footnotetext{
* Correspondence: 189962700@qq.com

${ }^{1}$ Anatomical Institute of Minimally Invasive Surgery, Southern Medical

University, Guangzhou 510515, China

Full list of author information is available at the end of the article
}

buttock pain and affected leg pain. The system is similar to nerve root pain associated with LDH.4 Therefore, it is important to make a definite diagnosis for correct treatment.

Previous studies have shown an association between radicular LBP and GP [3-7]. Studies locating the specific level responsible for gluteal pain in lumbar disc hernias have rarely been reported.

\section{Methods \\ Design}

All cases of LDH in the Kanghua hospital from 2010 to 2014 were recruited. An investigator who was blinded to LDH-related gluteal pain took the patients' histories and performed the physical examinations. Patients were evaluated for the presence or absence of gluteal pain and were allocated to a GP group and a non-GP group. All patients underwent a lumbar spine MRI to clarify their LDH diagnosis. To determine the cause and effect relationship 
between LDH and GP, percutaneous endoscopic lumbar discectomy (PELD) was performed to determine the relative presence of gluteal pain with $\mathrm{LDH}$. Patients were evaluated again for the presence or absence of gluteal pain after surgery.

\section{Inclusion and exclusion criteria}

The inclusion criteria were as follows: all LDH cases with PELD surgery to determine the relationship between gluteal pain and LDH. The cases in which gluteal pain disappeared after surgery were included. The exclusion criteria were as follows: we excluded patients with hip disease, piriformis syndrome, muscle strain, cancer, arthritis of the sacroiliac joints and multiple levels of LDH. The cases in which gluteal pain did not disappear after surgery were excluded.

\section{Follow-up evaluations}

Patients were followed up regularly by the operating surgeon for 24 months after their surgeries. The incidences of complications in the surgery groups, including the incidence of neurological complications and ODI improvement rates, were assessed.

\section{Data analysis}

The associations between level and gluteal pain and the incidence of neurological complications were evaluated in a $2 \times 2$ contingency table. ODI improvement rates were evaluated using a Mann-Whitney Test.

\section{Results}

From 2010 to 2014, 286 cases were included according to the inclusion criteria. Table 1 provided participant characteristics at baseline. Of these, 168 cases had gluteal pain (GP group) and 91cases had no pain in the gluteal area (non-GP group). Patients in the GP group ranged in age from 18 to 68 years, with a mean age of 39.7 years. Patients in the non-GP group ranged in age from 20 to 63 years, with a mean age of 41.0 years. All of the patients presented symptoms and confirmatory signs of lumbar radiculopathy that were consistent with the symptomatic disc level and MRI imaging findings.

Table 1 Patient demographic characteristics

\begin{tabular}{lll}
\hline & GP group $(n=168)$ & non-GP group $(n=118)$ \\
\hline Age & $39.7 \pm 9.6$ & $41.0 \pm 10.9$ \\
Gender (male: female) & $122: 46$ & $80: 38$ \\
Level & & \\
L3/4 & 0 & 27 \\
L4/5 & 159 & 43 \\
L5/S1 & 9 & 48 \\
\hline
\end{tabular}

Table 2 presented the association between GP and LDH. In the GP group, 159 cases involved the L4/5 level, accounting for $94.6 \%$, and 9 cases involved the L5/S1 level, accounting for $5.4 \%$. In the non-GP group, 43 cases involved the L4/5 level, and 48 cases involved the L5/S1 level. L4/5 level involvement accounted for $94.6 \%$ of the GP group, larger than the involvement of the L5/S1 level $(P<0.001)$, indicating that the $L 4 / 5$ level was a primary cause in gluteal-related LBP. No cases of gluteal pain involved the L3/4 level.

Percutaneous endoscopic lumbar discectomy surgeries were performed in the GP group. In both the GP and nonGP groups, the mean ODI values decreased significantly after surgery (from $69.3 \pm 8.1$ to $13.5 \pm 7.0, p<0.001$, and from $71.0 \pm 7.9$ to $15.1 \pm 7.9, p<0.001$, respectively). A total of $63.1 \%$ of the patients in the GP group felt no pain after surgery. One month after their surgeries, gluteal pain disappeared in all of the patients in the GP group, which indicated that LDH was responsible for gluteal pain. The pain appeared again in a patient with $\mathrm{L} 4 / 5$ disc recurrence, confirming the hypothesis.

With regard to the incidence of neurological complications in the GP group, there were 19 cases of lower limb numbness during surgery, accounting for $11.3 \%$ of the GP group and including two cases of foot drop and two cases of cerebrospinal leakage. These patients recovered 6-12 months after surgery. Regarding the incidence of neurological complications in the epidural anaesthesia group, there were 11 cases of lower limb numbness during surgery, accounting for $10.2 \%$ of the non-GP group and including one case of foot drop and two cases of cerebrospinal leakage. These patients recovered 6-12 months after surgery. No significant difference was observed regarding the incidences of neurological complications between the two groups $(P>0.05)$.

\section{Discussion}

Gluteal pain may derive from hip disease, piriformis syndrome, muscle strain, cancer and arthritis of the sacroiliac joints [8-10]. Because the symptoms are similar, LDHrelated gluteal pain can be misdiagnosed as superior cluneal nerve entrapment neuropathy (SCNEN) or muscle strain. Yasuhiro Chiba [3] reported five patients with intermittent LBP due to SCNEN who had previously received conservative treatment and who underwent surgery and

Table 2 Association between gluteal pain and lumbar disc hernia

\begin{tabular}{llll}
\hline & $L 3 / 4$ & $L 4 / 5$ & $L 5 / S 1$ \\
\hline GP group & 0 & $159(78.7 \%)$ & $9(15.8 \%)$ \\
Non-GP group & 27 & $43(22.3 \%)$ & $48(84.2 \%)$ \\
Statistical parameter & & & $X^{2}=77.23$ \\
$P$-value & & & $P<0.001$ \\
\hline
\end{tabular}


achieved good results. Adelmanesh F [4, 6] reported that trigger points in the superior-lateral quadrant of the gluteal area are highly specific indicators for radicular LBP. Thiese MS [5] found that patients with LBP had pain in the gluteal areas. All of the studies reported an association between radicular LBP and gluteal pain. However, the specific level responsible for gluteal pain in lumbar disc hernia has rarely been reported.

We could not completely rule out those patients with hip disease and musculoskeletal disease only by physical examination, electromyography and MRI. To avoid mixing the results and to identify the casual relationship between GP and LDH, PELD was induced to remove prominent nucleus pulposus, and GP was then re-evaluated after surgery. The disappearance of GP after surgery meant that the GP was caused by LDH. Only the cases in which GP disappeared after surgery were included in this study.

From this study, in the GP group, 159 cases involved the L4/5 level, accounting for $94.6 \%$; only nine cases involved the L5/S1 level, accounting for $5.4 \%$. Thus, the L4/5 level was a predominant culprit in glutealrelated LBP, and the results did not indicate a correlation between the L3/4 level and gluteal-related LBP. Among cases of L4/5 level involvement with lumbar disc herniation, $78.7 \%$ had gluteal pain, while among cases with L5/S1 level involvement with lumbar disc herniation, only $15.8 \%$ gluteal pain. No cases of L3/4 level involvement with lumbar disc herniation had gluteal pain.

All of the cases were subjected to percutaneous endoscopic lumbar discectomy, and gluteal pain subsided gradually post-surgery in all of the cases. Gluteal pain relapsed in one patient with L4/5 disc recurrence. This finding could imply a cause and effect relationship between LDH and gluteal pain. L4/5 disc hernia was a predominant factor in LDH-related gluteal pain.

PELD has become a standard procedure in recent years as a result of several advantages. Because PELD surgery only removes the hernia nucleus pulposus and does not disrupt the other structures of the lower back, the results are not confounded. Post-operative dysesthesia (POD) due to existing dorsal root ganglion (DRG) injury is a unique complication of PELD that accounts for the majority of neurological complications. As shown in Table 3, the two groups exhibited no significant differences with regard to incidences of neurological complications and ODI improvement rates.

The clinical implications of this study are as follows. Firstly, because the results suggest a clear relationship between L4/5 disc herniation and gluteal pain, GP assessment could help in diagnosing patients with L4/5 disc herniation. Secondly, beyond the accepted therapies aimed at the gluteal area, PELD may be suggested if
Table 3 Comparison of clinical results between the GP group and the non-GP group

\begin{tabular}{lll}
\hline & $\begin{array}{l}\text { Neurological } \\
\text { complications }\end{array}$ & $\begin{array}{l}\text { ODI improvement } \\
\text { rate }\end{array}$ \\
\hline GP group & $11.3 \%$ & $85.6 \%$ \\
Non-GP group & $10.2 \%$ & $85.8 \%$ \\
$\begin{array}{l}\text { Statistical } \\
\text { parameter }\end{array}$ & $\mathrm{X}^{2}=0.237$ & $\mathrm{Z}=0.633$ \\
P-value & 0.626 & 0.527 \\
\hline
\end{tabular}

the diagnosis of LDH is clarified, which might improve outcomes. This hypothesis should be tested in further anatomical studies.

\section{Conclusions}

This study is a retrospective study in which only the cases subjected to PELD surgery were included. Additionally, this study is not multi-centre. All of these factors may lead to biases. However, the goal of this study was to locate the specific level that was responsible for gluteal pain in $\mathrm{LDH}$. In this regard, we found that $\mathrm{L} 4 / 5$ disc hernias were a main factor in LDH-related gluteal pain. Further anatomical studies may be undertaken to support these results.

\section{Additional file}

Additional file 1: LDH_GP.sav. (SAV $31 \mathrm{~kb}$ )

Acknowledgements

This project was undertaken as part of an Undergraduate Research Fellowship at Southern Medical University in China. We acknowledge the staff of Kanghua hospital for their assistance on this study.

\section{Funding}

No funding was obtained for this study.

Availability of data and materials

The dataset supporting the conclusions of this article is available in the Additional file 1

Authors' contributions

DZH made substantial contributions to the conception, design and statistical analysis of the study. FGF was the main writer of the paper conceived the study idea and design, collected the data, clinical follow-up and interpretation of results. ZJH and XXY contributed to data collection and clinical follow-up. LYT contributed to the statistics of data and manuscript revision. SHx contributed to language corrections and interpretation of results. All authors read and approved the final manuscript.

\section{Competing interests}

The authors declare that they have no competing interests.

\section{Conflict of interest statement}

We declare that we have no financial and personal relationships with other people or organizations that can inappropriately influence our work, there is no professional or other personal interest of the manuscript entitled "Which level is responsible for gluteal pain in lumbar disc hernia?"

Consent for publication

Not applicable. 


\section{Ethics approval and consent to participate}

The Ethics Committee of Kanghua Hospital approved this study. All participants signed informed consent for using their data for the research purposes.

\section{Author details}

'Anatomical Institute of Minimally Invasive Surgery, Southern Medical University, Guangzhou 510515, China. ${ }^{2}$ Department of Orthopedics, Shenzhen Hospital of Southern Medical University, NO.1333, Xinhu Road, Shenzhen 518100, China. ${ }^{3}$ Spine Department of Kanghua Hospital, Dongguang city, Guangdong Province, China. ${ }^{4}$ Department of Anesthesia, Shenzhen Hospital of Southern Medical University, NO.1333, Xinhu Road, Shenzhen 518100, China.

Received: 1 March 2016 Accepted: 5 August 2016

Published online: 22 August 2016

References

1. Zhang YG, Guo TM, Guo X, et al. Clinical diagnosis for discogenic lower back pain. Int J Biol Sci. 2009;5:647-58.

2. Karas R, Mclntosh $\mathrm{G}$, Hall $H$, et al. The relationship between nonorganic signs and centralization of symptoms in the prediction of return to work for patients with lower back pain. Phys Ther. 1997:77:354-60.

3. Adelmanesh F, Jalali A, Jazayeri Shooshtari SM, Raissi GR, Ketabchi SM, Shi $Y$. Is there an association between Lumbosacral radiculopathy and painful Gluteal trigger points?: A cross-sectional study. Am J Phys Med Rehabil. 2015:94(10):784-91.

4. Park MS, Yoon SJ, Jung SY, Kim SH. Clinical results of endoscopic sciatic nerve decompression for deep gluteal syndrome: mean 2-year follow-up. BMC Musculoskelet Disord. 2016;17(1):218

5. Chiba Y, Isu T, Kim K, Iwamoto N, Morimoto D, Yamazaki K, Hokari M, Isobe M, Kusano M. Association between intermittent low-back pain and superior cluneal nerve entrapment neuropathy. J Neurosurg Spine. 2015;13:1-5.

6. Adelmanesh F, Jalali A, Shirvani A, Pakmanesh K, Pourafkari M, Raissi GR, Shi $Y$. The diagnostic accuracy of gluteal trigger points to differentiate radicular from non-radicular LBP. Clin J Pain. 2015;32:666-72. PMID:26491935.

7. Thiese MS, Hegmann KT, Wood EM, Garg A, Moore JS, Kapellusch J, Foster J, Ott U. Prevalence of LBP by anatomic location and intensity in an occupational population. BMC Musculoskelet Disord. 2014;15:283.

8. Laslett M, Young SB, Aprill CN, et al. Diagnosing painful sacroiliac joints: a validity study of a McKenzie evaluation and sacroiliac joint provocation tests. Aust J Physiother. 2003;49:89-97.

9. Hong C-Z. Muscle pain syndromes. In: Braddom RL, Chan L, Harrast MA, editors. Physical medicine and rehabilitation. Philadelphia: Saunders; 2011. p. 971.

10. Podschun L, Hanney WJ, Kolber MJ, Garcia A, Rothschild CE. Differential diagnosis of deep gluteal pain in a female runner with pelvic involvement: a case report. Int J Sports Phys Ther. 2013;8(4):462-71.

\section{Submit your next manuscript to BioMed Central and we will help you at every step:}

- We accept pre-submission inquiries

- Our selector tool helps you to find the most relevant journal

- We provide round the clock customer support

- Convenient online submission

- Thorough peer review

- Inclusion in PubMed and all major indexing services

- Maximum visibility for your research

Submit your manuscript at www.biomedcentral.com/submit
Biomed Central 